\title{
Molecular Imaging Training for Nuclear Medicine Residents
}

\author{
Michael M. Graham ${ }^{1}$ and Heather A. Jacene ${ }^{2}$ \\ ${ }^{I}$ Division of Nuclear Medicine, Department of Radiology, University of Iowa, Iowa City, Iowa; and ${ }^{2}$ Division of Nuclear Medicine, \\ Department of Imaging, Dana-Farber Cancer Institute, Boston, Massachusetts
}

$\mathbf{N}$ fecting the field are the advent of hybrid imaging, an increasing emphasis on evidence-based medicine, and a growing recognition of the potential of personalized medicine. Molecular imaging, which is a fundamental part of nuclear medicine, has an enormous potential to be a central part of the shift toward personalized medicine. Several new approaches and radiopharmaceuticals are emerging from basic science laboratories and are poised to become powerful tools in ensuring that patients receive optimal therapy for their disease and avoid ineffective treatment.

In 2006, the Society of Nuclear Medicine's Molecular Imaging Center of Excellence, renamed in 2010 as the Center for Molecular Imaging Innovation and Translation, established an education task force to examine the educational needs for current and future physicians and scientists practicing molecular imaging. This effort included curriculum development and expansion of the molecular imaging educational offerings and resources provided by the society. In September 2007 and May 2008, the task force submitted to the Nuclear Medicine Residency Review Committee of the Accreditation Council for Graduate Medical Education a set of recommendations for increasing the emphasis on molecular imaging instruction in nuclear medicine training. The new nuclear medicine residency program requirements that became effective in July 2011 contain a statement that residency programs must have didactic lecture topics including "fundamentals of imaging molecular targets, processes and events and existing and emerging molecular imaging techniques, particularly as they relate to current clinical practice." This was an important first step forward, but in our opinion it does not go far enough.

In 2011, the American Board of Nuclear Medicine published its position statement on nuclear medicine professional competency and scope of practice (1). This statement includes in section IX a requirement that practitioners of nuclear medicine have knowledge and understanding of

Received Feb. 2, 2012; revision accepted Feb. 14, 2012.

For correspondence or reprints contact: Michael M. Graham, University of lowa, 3863 JPP Department of Radiology, 200 Hawkins Dr., lowa City, IA 52242-1077.

E-mail: michael-graham@uiowa.edu

Published online Mar. 12, 2012.

COPYRIGHT (C) 2012 by the Society of Nuclear Medicine, Inc.

DOI: 10.2967/jnumed.112.103994 new molecular imaging probes and approaches under preclinical assessment. These include approaches to identification of targets for molecular imaging, development of new molecular imaging probes and strategies, testing and validation of new imaging tracers for molecular targets, reporter gene strategies, and regulatory requirements for clinical translation of new molecular imaging agents.

If these novel methods of molecular imaging are to be incorporated into the clinical practice of nuclear medicine, then nuclear medicine residents, physicians, and academic faculty must become familiar with them. If training is constrained to teaching only what is in current practice, then progress becomes severely inhibited. Nuclear medicine physicians and radiologists must embrace the breadth of molecular imaging, including nonradioactive methods. We have to become familiar with these methods, and they have to be included as part of nuclear medicine resident education.

One obstacle in moving forward is that many of these technologies are performed at the preclinical and translational levels but are not yet in common clinical practice. A similar situation happened when MRI was introduced. This was an entirely new method that had not been covered in prior radiology training programs. However, it was so clear that MRI had enormous potential that a major educational effort was established at various levels appropriate for practicing radiologists, faculty, and residents, and a successful transition was made to incorporate MRI into radiology. The challenge with molecular imaging is that its enormous potential may not be as well understood, and molecular imaging may therefore take longer than desirable to become a central part of clinical medicine. Nevertheless, molecular imaging methods are rapidly developing, and it is essential that nuclear medicine physicians in training become familiar with them. Clinical medicine is also rapidly progressing. The molecular bases of diseases are being further elucidated, and highly specific targeted therapies are being developed. Nuclear medicine physicians need to be aware of these advances as well and need to be able to "speak the same language" as their clinical colleagues in order to optimize the use of these moleculetargeted therapeutic agents in imaging.

Many nuclear medicine training programs have an opportunity to lead the effort in teaching their residents the basics of molecular imaging. The suggestions presented 
in the curriculum below are the essence of those originally made to the Nuclear Medicine Residency Review Committee. Some or all of these suggestions can be integrated into training programs. This curriculum will allow the nuclear medicine community to create a large group of nuclear medicine physicians with skills in molecular imaging. We strongly believe that incorporating this curriculum will change the practice of nuclear medicine so that we will be in a position to use the tools of molecular imaging effectively in the future. The principles and topics described here should be part of training programs for all residents who will be practicing nuclear medicine.

Aside from a few agents, nuclear medicine is molecular imaging. Many of the agents that we use depict various aspects of metabolism or the distribution of receptors. The newer approaches of molecular imaging are straightforward applications and extensions of what we are already doing.

Because molecular imaging is such a central part of nuclear medicine, many existing program requirements are already appropriate for a curriculum that supports training in molecular imaging and overlap significantly with the suggestions below. These suggestions are not intended to cover all program requirements, nor do they exhaustively cover all of molecular imaging.

1. Closely follow scientific progress in nuclear medicine and molecular imaging, and learn to incorporate it effectively for modifying and improving diagnostic and therapeutic procedures.

2. Become familiar with and regularly read the major journals in nuclear medicine and molecular imaging. During residency this will involve regular participation in a journal club.

3. Understand and use quality control procedures for imaging devices, laboratory instrumentation, radiopharmaceuticals, and nonradioactive molecular imaging agents.

4. Receive didactic instruction in the following areas:

a. The principles of instrumentation used in detection, measurement, and imaging of radioactivity, with special emphasis on $\gamma$-cameras, SPECT and PET devices, and associated electronic instrumentation and computers used in image production and display. Instruction should include the instrumentation principles involved in hybrid imaging, MRI, spectroscopy, ultrasound, multislice CT, optical imaging of bioluminescence and fluorescence, and small-animal imaging instrumentation.

b. Mathematics, statistics, and computer sciences as applied to imaging: probability distributions; applications of mathematics to tracer kinetics, including compartmental modeling and quantification of physiologic processes; demonstration of a working knowledge of computational image processing, which may include interactive processing of images, including algorithms for attenuation and scatter corrections, quality control of image-acquisition, and processing. c. Medical decision making with an emphasis on efficacy of imaging.

d. Molecular and cellular biology: the imaging of molecular targets, processes, and events, including the basic principles of molecular and cellular biology. Examples of imaging molecular and cellular processes, along with imaging agents, are not limited to but include the following:

i. Metabolism (existing agents such as biliary agents, bone agents, renal agents, iodocholesterol, and ${ }^{18} \mathrm{~F}-$ FDG and emerging agents such as acetate, choline, FDOPA, FACBC, and amino acids).

ii. Cellular proliferation (thymidine, FLT, and FMAU).

iii. Receptors (existing agents such as octreotide, MIBG, depreotide, and ${ }^{123}$ I-FP-CIT [DaTscan; GE Healthcare]) and emerging agents such as DOTATOC, DOTATATE, melanocyte-stimulating hormone, bombesin, and FDHT).

iv. Amyloid imaging agents (emerging agents such as florbetapir, florbetaben, and flutemetamol).

v. Antibodies (existing agents such as capromab, ibritumomab, and tositumomab and emerging agents such as renal cell carcinoma-associated antigen G250 [CAIX antibody]).

vi. Hypoxia (FMISO, ATSM), FAZA, EF-5, and HX-4).

vii. Reporter genes (NaI symporter, somatostatin receptor, and herpes simplex virus thymidine kinase).

viii. Apoptosis (annexin V and ML-10).

ix. Angiogenesis (RGD-based agents).

x. Cell trafficking (direct labeling [e.g., $\left.{ }^{111} \mathrm{In}\right]$ and indirect labeling [e.g., reporter genes]).

xi. Optical imaging (bioluminescence [e.g., luciferin and luciferase] and fluorescence, including near infrared [e.g., indocyanine green]).

Residents should be familiar with the established radiopharmaceuticals, as well as with emerging molecular imaging agents, including those that are nonradioactive. Residents should also understand how these agents are used for diagnosis, for staging, and for selecting and monitoring the efficacy of therapy. The emerging concept of theranostics, which pairs suitable diagnostic and therapeutic agents, will also need to be recognized. The quantitative use of molecular imaging agents builds on an understanding of targeting and pharmacokinetic analysis (e.g., compartmental modeling).

e. Aspects of radionuclide production and radiopharmaceutical design, synthesis, and biologic evaluation: reactor, cyclotron, and generator production of radionuclides; radiochemistry; pharmacokinetics; and formulation of radiopharmaceuticals. These aspects include basic strategies for radiolabeling small molecules, peptides, antibodies, aptamers, and biomolecules 
with radionuclides for SPECT and PET, with emphasis on chelation strategies appropriate for each radionuclide-molecule pair, as well as labeling with radiohalogens (fluorine, bromine, and iodine radionuclides). Also included should be an overview of radionuclides relative to molecular therapies and strategies used to increase radiotherapeutic targeting, including multistep targeting approaches.

f. Nonradioactive agents (e.g., for optical imaging, ultrasound, MRI, and CT) such as fluorescent dyes and proteins (including near-infrared), microbubbles, nanoparticles, and contrast agents, and applications of certain classes of molecular agents for therapeutic purposes (e.g., photodynamic therapy).

Residents should have continuing instruction in the relevant basic sciences. This should include formal lectures and formal labs, with an appropriate balance of time allocated to the major subject areas, which should include physical science and instrumentation, radiation biology, radiation protection and regulatory issues, molecular and cellular biology, and molecular imaging agents. Instruction in the basic sciences should not be limited to didactic sessions. The resident's activities should also include laboratory experience and regular contact with basic scientists in their clinical adjunctive roles.

It is important that a program director contemplating the above suggestions should not view them as an all-or-none situation. It is unlikely that any program will have the resources to immediately implement all the suggestions, and it is likely that several programs already provide many of these topics in their current curricula. The goal is to provide program directors with the elements needed to incorporate molecular imaging into nuclear medicine residency programs so that the transition can get under way.
In addition, these suggestions are not being made with the expectation that all program directors need to gather the necessary teaching material on their own. The Center for Molecular Imaging Innovation and Translation has made a major effort to compile much of the necessary teaching material, including scientific papers (particularly reviews), PowerPoint presentations, videos, and webinars. The Society of Nuclear Medicine recently released a 13-part training webinar series on the topics described above. On the Web site (www.snm.org/programdirectors), the Center for Molecular Imaging Innovation and Translation has provided a compendium of resources for trainees and others who want to learn more about the principles of molecular imaging. Finally, we are working with Cambridge University Press to publish a book outlining these critical principles that will prepare residents to embrace the latest molecular imaging innovations.

Current and forthcoming applications in molecular imaging promise to have a significant impact on the future of clinical care for patients. Including education on more advanced molecular imaging processes and techniques in nuclear medicine residency training programs is critical to ensuring that the field continues to advance and meets the future needs of patient care, including personalized medicine. The suggestions presented here represent the initial steps that programs should consider and use to enhance trainee exposure to advanced molecular imaging.

\section{ACKNOWLEDGMENT}

This document was endorsed by the Board of Directors of the Society of Nuclear Medicine on January 28, 2012.

\section{REFERENCE}

1. Frey KA, Royal HD, Di Carli MF, et al. ABNM position statement: nuclear medicine professional competency and scope of practice. J Nucl Med. 2011;52:994-997. 